\title{
Electroencephalographic Response to Procedural Pain in Healthy Term Newborn Infants
}

\author{
ELISABETH NORMAN, INGMAR ROSÉN, SAMPSA VANHATALO, KARIN STJERNQVIST, OVE ÖKLAND, \\ VINETA FELLMAN, AND LENA HELLSTRÖM-WESTAS
}

\begin{abstract}
Department of Pediatrics [E.N., V.F., L.H.-W.], Department of Clinical Neurophysiology [I.R.], Lund University Hospital, Lund SE-22185, Sweden; Department of Clinical Neurophysiology [S.V.], Department of Pediatrics [V.F.], University Hospital of Helsinki, Helsinki FIN-00029, Finland; Department of Psychology [K.S.], Lund University, Lund SE-22100, Sweden; Department of Pediatrics [O.O.], Ålesund Hospital, Ålesund N-6026, Norway; Department of Women's and Children's Health [L.H.-W.], Uppsala University, Uppsala SE-75185, Sweden
\end{abstract}

\begin{abstract}
The current study aimed to characterize changes in EEG-related measures after noxious stimuli in neonates and to assess their potential utility as measures of pain and/or discomfort during neonatal intensive care. Seventy-two healthy term infants were investigated: Twenty-eight had a non-skin-breaking pin-prick on the heel, randomized to receive either oral glucose $(n=16)$ or water ( $n=12$ ) before the stimulus. Twenty-one infants were studied during a venous blood sample from the dorsum of the hand, 23 infants during a capillary heel stick. Behavioral pain responses were assessed with the Premature Infant Pain Profile Scale. The stimulus evoked a significant increase in higher frequency components $(10-30 \mathrm{~Hz})$ which also correlated to behavioral measures. The frontotemporal localization of the increased activity with frequency bands similar to electromuscular artifacts and the relation to behavioral measures confirmed that this activity corresponds to an increase in muscle tone. There was no change in frontal EEG asymmetry in any of the groups. The present results indicate that responses in cortical activity recorded by EEG are not useful for clinical assessment of infants' responses to noxious stimuli. (Pediatr Res 64: 429-434, 2008)
\end{abstract}

$\mathrm{N}$ ewborn infants undergoing intensive care may become exposed to a considerable number of painful experiences (1-3). Identification and assessment of pain in sick newborn infants is challenging because the infant's ability to communicate pain is limited. Pain assessment instruments for clinical use have mainly been constructed for evaluation of acute procedure-related pain responses (4). There is a growing international request to produce valid instruments for assessing both continuous and procedure-related pain in newborns $(5,6)$.

Although the somatic sensation of painful stimuli undoubtedly takes place within hundreds of milliseconds after the stimulus, several studies have demonstrated that painful stimuli also produce more long-lasting behavioral and physiologic responses $(7,8)$. A previous study indicated that repeated heel strokes from the Neonatal Behavioral Assessment Scale (9) in healthy term infants resulted in a change in the symmetry of frontal EEG activity within the theta frequency band (3-6

Received November 19, 2007; accepted May 12, 2008.

Correspondence: Elisabeth Norman, M.D., Neonatal Intensive Care Unit, University Hospital, SE-22185 Lund, Sweden; e-mail: elisabeth.norman@med.lu.se

Supported by the Swedish Medical Research Council [VR grant nos. 0037 (L.H.W.), 0084 (I.R.)] and ALF and Slättens (V.F.), from Arvo and Lea Ylppo Foundation and Juselius Foundation (S.V.) and from Lund University.
$\mathrm{Hz}$ ), and this change was attenuated by oral sucrose given before the noxious stimulus (10). Another study showed that in 10-mo-old infants, EEG "resting asymmetry" correlated with behavioral responses when infants were separated from their mothers (11). The investigators suggested that an infant's distress response may be predicted from prior measures of baseline frontal EEG asymmetry. In adults, "frontal EEG asymmetry" has been widely used as a neurophysiologic marker of emotional reactions in various psychological studies (12).

Very little is known about EEG responses to pain in newborn infants. The option of using a relatively simple EEG measure of distress in neonates is clinically intriguing because it could be readily implemented as a trend display in continuous EEG monitoring of neonates. Thus, the objective of the present study was to characterize potential changes in the EEG after acute noxious or painful stimuli in healthy term infants and to evaluate their utility for pain assessment.

\section{METHODS}

Patients. Three groups of healthy term infants were investigated at Lund University Hospital, Sweden and Ålesund Hospital, Norway, during the time-period March 2004 to March 2006. Inclusion criteria were term birth (gestational age, $37 \mathrm{wk}$ or more), normal pregnancy and normal delivery (vaginal or elective caesarean section), 5-min Apgar score 7 or more, a birth weight appropriate for gestational age, and no postnatal disorder. Exclusion criteria were ongoing maternal or infant analgesic treatment.

Group 1 consisted of 31 term infants who were recruited at the maternity unit and had a full EEG (electrode positions according to the International 10-20 system: F3, F4, Cz, P3, P4, C3, C4, T3, T4) recorded at the Department of Clinical Neurophysiology, Lund University Hospital (Nervus 3.3 EEG system with M40 amplifier, Viasys, Nicolet Biomedical, Madison, WI). The infants were randomized to receive either $1 \mathrm{~mL}$ of water (group 1a) or 1 $\mathrm{mL}$ glucose $300 \mathrm{mg} / \mathrm{mL}$ (group $1 \mathrm{~b}$ ) orally 2 min before a brief noxious, but not skin-breaking, "pin-prick" on either the left or the right foot. The pin-prick procedure was performed with a wooden toothpick, pressed distinctly during $1 \mathrm{~s}$ toward the central part of the sole of the heel according to the Neonatal Behavioral Assessment Scale (9). The randomization (sealed envelopes) and preparation of the solution was performed blindly.

Groups 2 and 3 had two different blood sampling procedures performed according to local routines for standard metabolic screening. In group $2 \mathrm{a}$, a venous blood sample from the dorsum of the left hand was obtained from 22 infants at an age of $72 \mathrm{~h}$ or more at the maternity unit at Lund University Hospital, all by the same experienced midwife. In group $3(n=25)$, capillary

\footnotetext{
Abbreviations: aEEG, amplitude-integrated electroencephalogram; EMG, electromyogram; FFT, Fast Fourier Transformation; PIPP, Premature Infant Pain Profile Scale; TF, time-frequency
} 
Table 1. Clinical characteristics for the three groups of infants

\begin{tabular}{|c|c|c|c|c|}
\hline Group & 1a & $1 \mathrm{~b}$ & 2 & 3 \\
\hline Study characteristics & Heel-prick, water & Heel-prick, glucose & $\begin{array}{l}\text { Venous blood sample } \\
\text { hand }\end{array}$ & $\begin{array}{l}\text { Capillary heel-lancet } \\
\text { blood sample }\end{array}$ \\
\hline Number of infants & 12 & 16 & 21 & 23 \\
\hline Sex (male/female) & $6 / 7$ & $8 / 7$ & $12 / 9$ & $10 / 13$ \\
\hline GA, wk & $40(39-42)$ & $39.5(37-41)$ & $40(38-41)$ & $40(37-41)$ \\
\hline PNA at EEG, $h$ & $67.5(42-84)$ & $56.5(37-94)^{*}$ & $120(96-143)^{*}$ & $70(61-81)$ \\
\hline Awake/asleep before stimulus, $n$ & $8 / 4$ & $11 / 5$ & $16 / 5$ & $13 / 10$ \\
\hline PIPP score & $11(0-14)^{* *}$ & $6(0-13) \dagger$ & $11.5(2-14)$ & $12(3-16)$ \\
\hline Stimulus left/right, $n$ & $7 / 5$ & $10 / 16$ & $21 / 0$ & $0 / 23$ \\
\hline EEG channels & $\begin{array}{l}\mathrm{F} 3, \mathrm{~F} 4, \mathrm{Cz}, \mathrm{P} 3, \mathrm{P} 4, \mathrm{C} 3 \\
\mathrm{C} 4, \mathrm{~T} 3, \mathrm{~T} 4\end{array}$ & $\begin{array}{l}\mathrm{F} 3, \mathrm{~F} 4, \mathrm{Cz}, \mathrm{P} 3, \mathrm{P} 4, \mathrm{C} 3 \\
\mathrm{C} 4, \mathrm{~T} 3, \mathrm{~T} 4\end{array}$ & $\mathrm{~F} 3, \mathrm{~F} 4, \mathrm{Cz}, \mathrm{P} 3, \mathrm{P} 4$ & $\mathrm{~F} 3, \mathrm{~F} 4, \mathrm{Cz}, \mathrm{P} 3, \mathrm{P} 4$ \\
\hline
\end{tabular}

Values are numbers, median, and ranges.

GA, gestational age in weeks; PNA, postnatal age in hours.

$* p<0.01$ for difference in PNA between groups $1 \mathrm{~b}$ and 3 , and between groups 2 and 3 .

$* * p<0.05$ between groups $1 \mathrm{a}$ and $1 \mathrm{~b}$.

$\dagger p<0.01$ between groups $1 \mathrm{~b}$ and 3 .

blood sampling was performed with an automatic lancet (Tenderfoot, Skafte Medlab AB, Onsala, Sweden) by laboratory staff at the maternity ward, Ålesund Hospital, Norway. The blood sample was taken from the right heel at a postnatal age of at least $60 \mathrm{~h}$. The EEGs in groups 2 and 3 were recorded from five derivations (F3, F4, Cz, P3, and P4) (Nervus monitor 1.3, Viasys, Nicolet Biomedical, Madison, WI). Clinical data are presented in Table 1.

In all the three groups, $\mathrm{Ag} / \mathrm{AgCl} \mathrm{EEG}$ disc electrodes were attached after skin preparation with an abrasive gel (Nuprep, D.O.Weaver \& Co., Aurora, Colorado) and a conductive paste (Ten20 Conductive EEG paste, D.O.Weaver \& Co., Aurora) and fixed with an elastic dressing fixation (Carefix Head, Tytex Group, Ikast, Denmark). The EEG signal was recorded with a sampling range of $256 \mathrm{~Hz}$ and with high pass filter at $0.10 \mathrm{~Hz}$ and low pass filter at $500 \mathrm{~Hz}$.

Written informed consent was obtained from all parents. The Regional Ethics Committees in Lund and at Trondheim University, respectively, approved the research protocols.

Study procedures. The mothers were encouraged to feed their babies before the examination. In group 1, electrode attachment took place 45-60 min before the stimulus, and in the other two groups approximately $10 \mathrm{~min}$ before the stimulus. First, at least 2 min of EEG was recorded when infants were in a calm stable state, asleep, or awake. Then the infants in group 1 received their allocated treatment, followed by another $2 \mathrm{~min}$ of registration before the noxious stimuli. The EEG response to the stimulus was recorded for at least $2 \mathrm{~min}$ in all groups.

Pain responses were assessed with the Premature Infant Pain Profile (PIPP) Scale (13). Oxygen saturation $\left(\mathrm{SaO}_{2}\right)$ and heart rate were monitored (Nellcor N395, Tyco Healthcare Norden AB, Sweden) and videotaped.

$\boldsymbol{E E G}$ analysis. All EEG registrations were assessed for artifacts by the first author (E.N.) and, when in doubt, also by a clinical neurophysiologist (I.R.), both blinded for group allocation. Six recordings (three in group 1, one in group 2, and two in group 3) were excluded because of technical artifacts, thus in total 72 recordings $(12+16$ in group 1, 21 in group 2 and 23 in group 3$)$ were included. The quantitative EEG analysis was performed from epochs consisting of 2 min before and after the painful stimulus, as well as $2 \mathrm{~min}$ before and after glucose/water administration.

Frequency domain analysis was performed by Fast Fourier Transformation (FFT); the changes in spectral characteristics (by wavelets) and amplitudeintegrated EEG (aEEG) properties analyzed over time. Such an approach is mathematically more challenging, but it makes it possible to assess without $a$ priori limitations which time and frequency window(s), if any, would be optimal for seeing the responses to the present stimuli.

Time series analyses. After digital filtering $(0.5-30 \mathrm{~Hz})$, the frequency domain characteristics were first analyzed by calculating FFT spectra for the absolute power $\left(\mu \mathrm{V}^{2}\right)$ over $10 \mathrm{~s}$ epochs (Hamming filter) averaged over $30 \mathrm{~s}$ artifact-free periods. The FFT spectra were divided into frequency bands: delta $0.5-3 \mathrm{~Hz}$, theta $4-7 \mathrm{~Hz}$, alpha $8-12 \mathrm{~Hz}$, and beta $13-30 \mathrm{~Hz}$, and a $3-6$ $\mathrm{Hz}$ band comparable to the study by Fernandez et al. (10). Higher frequencies than $30 \mathrm{~Hz}$ were not studied as neonatal cortical EEG is essentially limited to activity below $30 \mathrm{~Hz}(14)$. The infraslow and lowest delta frequencies (15) were omitted from the analysis, as they cannot be reliably extracted from the movement artifacts in our recording settings.

Ratios for absolute power in pre- versus post-stimulus epochs were calculated for all frequency bands (see above), and a frontal asymmetry index was created from natural logarithms for the absolute power within the 3-6 Hz band in the
F4- $\mathrm{Cz}$ and $\mathrm{F} 3-\mathrm{Cz}$ derivations by subtracting the natural logarithm for the band power on the left side from that on the right side ( Ln right - Ln left) (10).

Wavelet transformation was used to produce a detailed time-frequency (TF) analysis for frequencies from 2 to $32 \mathrm{~Hz}$ with Morlet wavelets $(m=6.5$, i.e., length of wavelet used for creating the graphs, frequency separation $1 \mathrm{SD}$ ) in a custom-made software package that runs under Labview program (16). Before wavelet transformation, the signals were exported into 180-s long epochs ( $60 \mathrm{~s}$ before and $120 \mathrm{~s}$ after the stimulus), that were sampled from 256 $\mathrm{Hz}$ to $64 \mathrm{~Hz}$ to reduce the computing load without meaningful compromise to the analysis. The amount of activity at specific high-frequency bands were quantified by taking the signal envelope of the traces that were obtained by combining wavelets within the given frequency band (16). All wavelet-based $\mathrm{TF}$ analyses were performed for each individual and averaged for the whole group. For the TF graphs, the averaged activity at each frequency during $60 \mathrm{~s}$ before the stimulus was subtracted from the averaged values obtained during $120 \mathrm{~s}$ after the stimulus.

aEEG. The aEEG amplitude shows a weighted representation of the amplitude of EEG frequencies between 2 and $15 \mathrm{~Hz}$ and uses a heavily compressed time scaling to enable evaluation of long-term changes and trends in electrocortical background activity (17-19).

For assessing the utility of the aEEG paradigm in evaluating pain responses, we exported the minimum and maximum aEEG amplitudes to excel-files with a time resolution of $4 \mathrm{~s}$, which were then used to calculate and plot both individual traces and group variables (mean \pm SEM).

Statistics. The absolute EEG power and the asymmetry index were analyzed using SPSS 14.0 software package and nonparametric tests, including bivariate correlations (Spearman) and paired statistics (Wilcoxon signed ranks test). In the wavelet analysis, the baseline epoch of $60 \mathrm{~s}$ before stimulus was used to assess the absolute change (simple subtraction of the average baseline from the response) or the parts of response that exceeded statistical significance ( $t$ test between baseline and response). The values of selected frequency bands as a function of time for each individual, and for the mean and its \pm SEM were calculated to better visualize the behavior over time of these values. Two-sided tests were used, and a $p<0.05$ was considered statistically significant.

Logistic regression analyses were used to investigate effects of the following background factors on EEG responses: group assignment, gestational age, postnatal age, and gender.

\section{RESULTS}

Behavioral response. The different stimuli all resulted in clear pain reactions, such as tachycardia, increased muscle activity, crying, and increased PIPP scores. The infants in group 1 who received glucose had lower PIPP scores than those given water $(p=0.048)$, and lower PIPP scores than infants in group 3 who underwent heel-lancet sampling $(p=0.004)$ (Table 1). Twenty-four infants were asleep when the stimulus was applied, and 48 were awake. There was no difference in PIPP scores $(p=0.239)$ between sleeping infants and those who 
were awake. Furthermore, PIPP scores did not differ between boys and girls.

EEG analyses-general characteristics of the EEG response. Infants who were sleeping before the stimulus had significantly more theta activity than infants who were awake; median (range) values for the $\mathrm{F} 3-\mathrm{Cz}$ signal were $11.1 \mu \mathrm{V}^{2}$ (4.2-32.3) in sleeping infants versus $8.5 \mu \mathrm{V}^{2}(4.7-46.6)$ in awake infants $(p=0.007)$, with corresponding values for the F4-Cz signal 13.1 $\mu \mathrm{V}^{2}$ (4.9-34.1) versus $10.1 \mu \mathrm{V}^{2}$ (5.3-45.3) $(p=0.042)$, respectively. There were no other EEG differences between sleeping and awake infants before the stimulus,

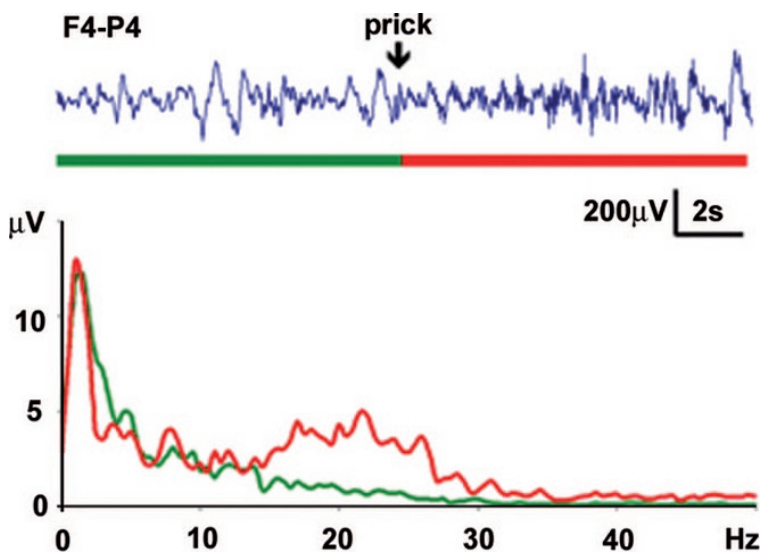

Figure 1. Example of spectral properties of EEG signal with and without EMG contamination in a newborn infant. The graph shows $20 \mathrm{~s}$ of EEG (F4-P4) before (green) and after (red) a noxious stimulus, which results in a robust increase in high-frequency activity, typical of muscle artifact (thick, noisy signal in the EEG above the red horizontal line). In the spectral analysis below, it becomes evident that the period of EEG with EMG activity has a distinct hump at $10-30 \mathrm{~Hz}$, representing muscle activity. neither for the 3-6 Hz activity nor for the other frequency bands or the baseline asymmetry index. The FFT and TF analyses of the EEG responses showed that the only sustained and statistically significant change in the EEG activity after the stimulus took place at frequencies between 10 and $30 \mathrm{~Hz}$, corresponding to the traditional beta band (Fig. 1). By comparing TF plots for all EEG derivations in group 1, it was clear that the increased $10-30 \mathrm{~Hz}$ activity was concentrated to the frontal and temporal areas, which are well known to be the sites of most muscular artifacts (data not shown).

The increase in 10-30 Hz activity was clearly larger in the babies that were awake compared with those who were asleep before and/or after the stimulus, as shown in Figure $2 A$ and $B$ from infants in group 3.

EEG asymmetry. Group 3 had a small baseline asymmetry within the theta frequency band $(3-6 \mathrm{~Hz})$ already before the stimulus, with a median left-sided power $9.1 \mu \mathrm{V}^{2}$ (range, 3.5-21.4) versus right-sided $11.8 \mu \mathrm{V}^{2}$ (range, 4.2-26.9), $p=$ 0.023 (Fig. 3). However, the noxious stimulus did not result in any significant change in EEG asymmetry in any of the groups for any of the frequency bands. In group 1, there were no differences between infants who received either oral glucose or water. There were no differences or changes in EEG asymmetry indices depending on whether the infants were asleep or awake before the stimulus.

There is a possibility that a transient laterality in the EEG could be too brief in duration to be seen when analyzing $30 \mathrm{~s}$ time windows with the FFT method. To clarify this, we produced subtraction images (left-right) of the TF representation of activity from the frontocentral and centrotemporal derivations in group 1. There was no lateralization of EEG
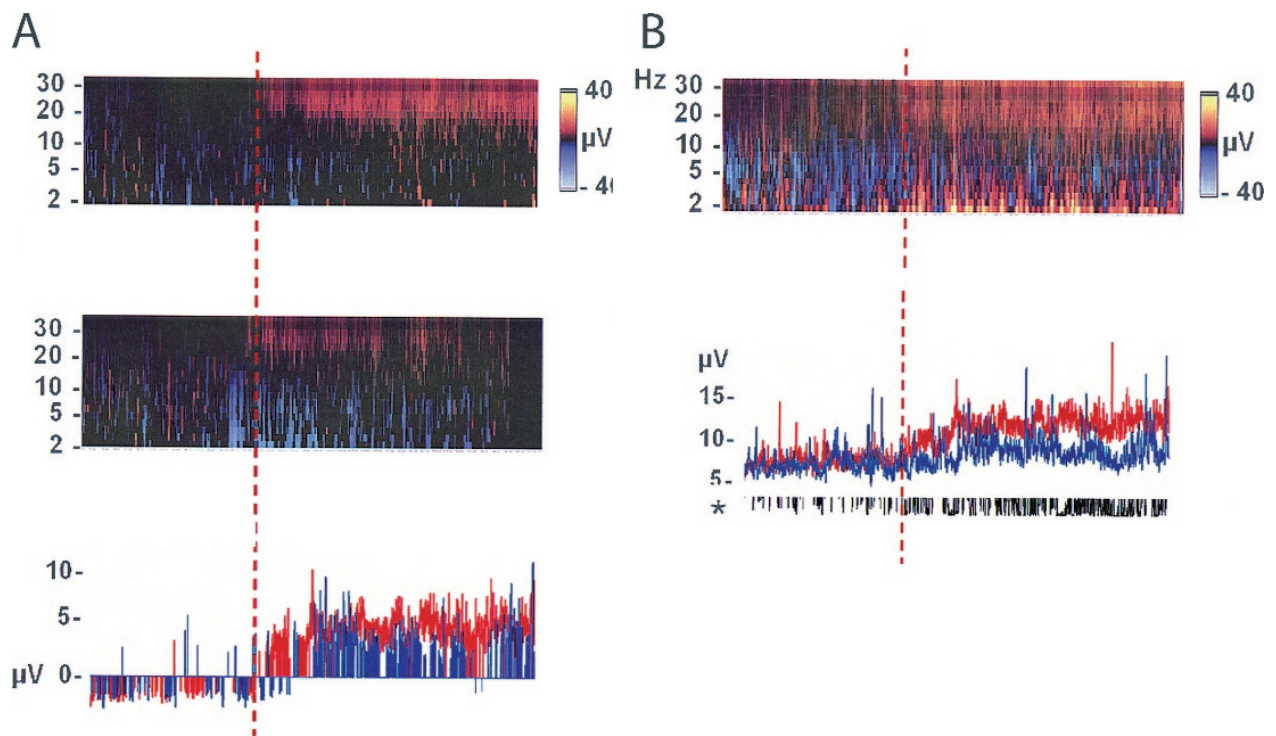

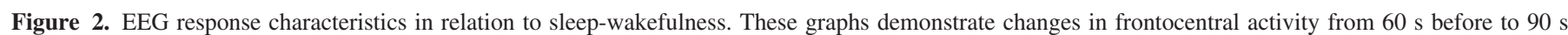

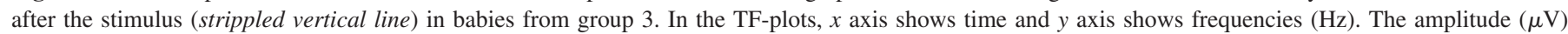

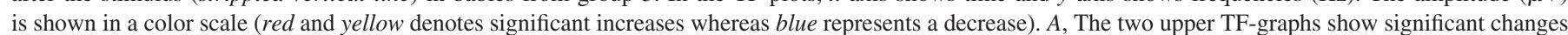

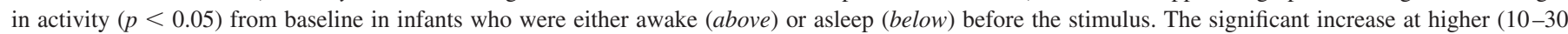

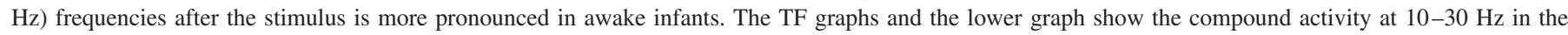

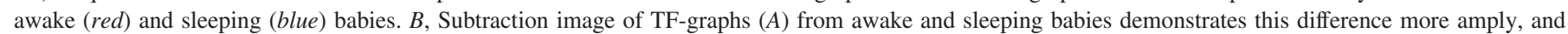

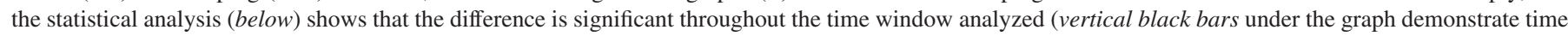
points at which the difference between sleeping and awake babies was significant). 


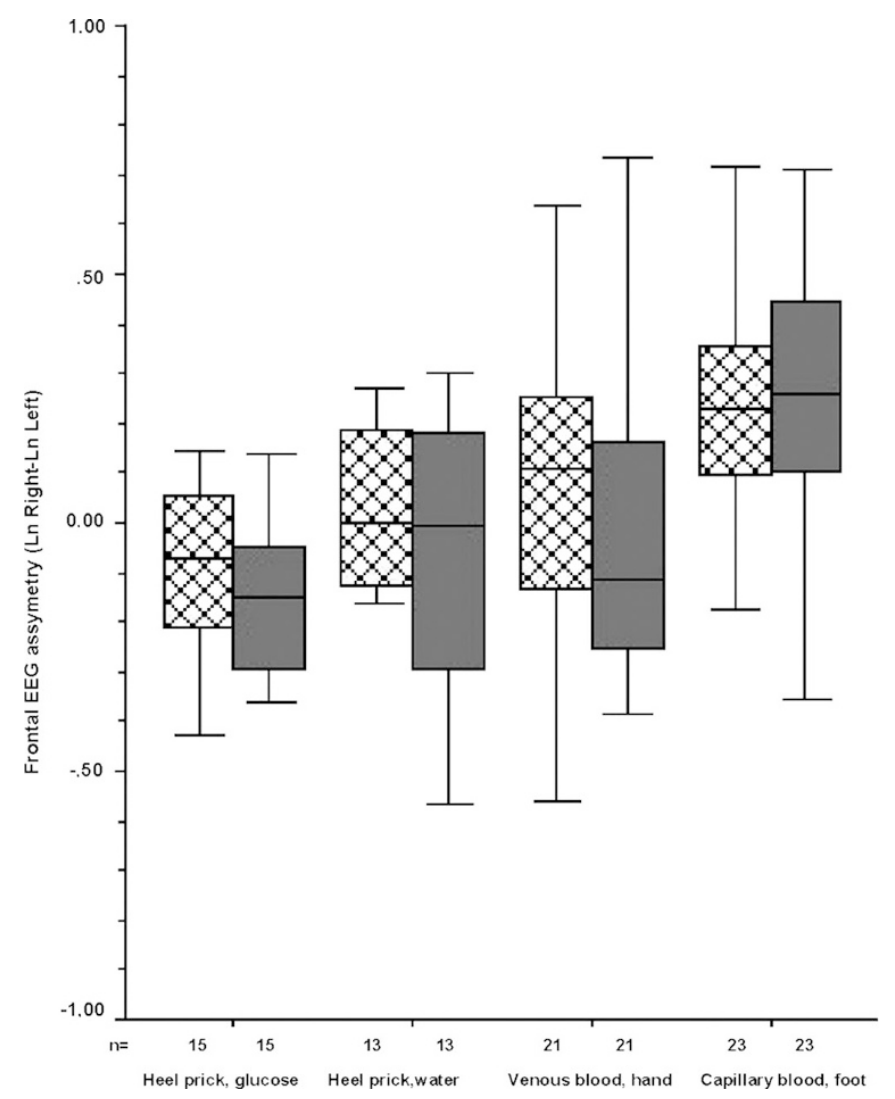

Figure 3. Frontal asymmetry indices. Boxplots representing frontal asymmetry indices, for 3-6 Hz activity, before (squared) vs after (dark) the stimulus for each group of infants. Infants in group 3 (capillary blood sampling from the foot) had a certain frontal asymmetry both before and after the stimulus, but there were no significant changes in laterality of the EEG responses from the stimulus in any of the groups.

activity neither between the left versus the right hemisphere nor between the contralateral versus the ipsilateral hemisphere, relative to the stimulus (Fig. $4 A$ and $B$ ).
Other observations related to the background variable versus $\boldsymbol{E E G}$ asymmetry. The postnatal or gestational age did not correlate to the EEG power measures.

The baseline 3-6 Hz asymmetry index was slightly higher in boys, median (range) 0.16 ( -0.31 to 0.72 ) compared with girls $0.04(-0.98$ to 1.07$)(p=0.046)$. There were no gender differences in the EEG response (before/after) to the noxious stimulus, neither as group differences nor as individual responses (evaluated by paired statistics). Logistic regression analyses showed that the asymmetry indices before the noxious stimulus were independently associated with infant's gender $(p=0.006)$, group assignment $(p<0.001)$, and postnatal age in hours $(p=0.033)$, but not with gestational age. However, none of these factors were associated with changes in the asymmetry index (before versus after the stimulus).

Pain responses in the aEEG trend. Figure 5 shows that there were no consistent changes in the aEEG trends before versus after the stimulus, occasional changes in the aEEG levels reflect movement artifacts and muscular activity.

Relation between behavioral and EEG responses. There was a significant positive correlation between behavioral pain expression (i.e., PIPP score) and the EEG power at higher frequency ranges in all channels. The correlations were strongest and most consistent within the beta frequency range in the frontocentral: $\mathrm{F} 3-\mathrm{Cz}\left(r_{\mathrm{s}}=0.411, p<0.001\right)$ and $\mathrm{F} 4-\mathrm{Cz}\left(r_{\mathrm{s}}=\right.$ $0.405, p<0.001)$; and in the frontoparietal leads: F3-P3 $\left(r_{\mathrm{s}}=\right.$ $0.543, p<0.001)$ and F4-P4 $\left(r_{\mathrm{s}}=0.486, p<0.001\right)$.

There were significant positive correlations between PIPP scores and poststimulus increases in EEG power at higher frequency ranges in all channels. The correlations were strongest and most consistent within the beta frequency range in the frontocentral: F3-Cz $\left(r_{\mathrm{s}}=0.404, p<0.001\right)$ and F4-Cz $\left(r_{\mathrm{s}}=\right.$ $0.413, p<0.001)$; and in the frontoparietal leads: F3-P3 $\left(r_{\mathrm{s}}=\right.$ $0.541, p<0.001)$ and F4-P4 $\left(r_{\mathrm{s}}=0.488, p<0.001\right)$.
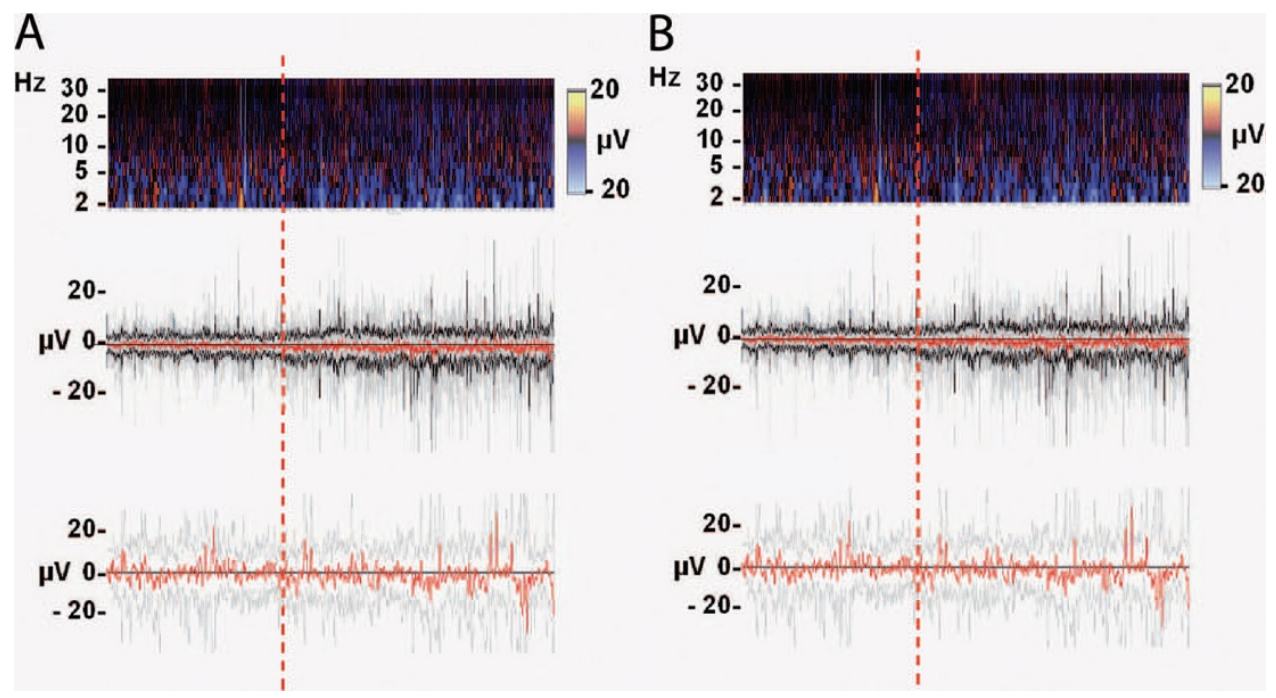

Figure 4. Lateralization of EEG responses. A, TF-representations of frontal EEG activity (left minus right hemisphere) from $60 \mathrm{~s}$ before to $90 \mathrm{~s}$ after the heel-prick procedure (vertical line) in group 1 show that there are no consistent changes in EEG lateralization as a response to the stimulus. The power values from the TF graph at 10-30 Hz (middle) and 3-6 Hz (below) show data for each individual baby (gray lines), the group average (red lines), and the SEM (black lines, only shown for $10-30 \mathrm{~Hz}$ activity). $B$, The two upper TF-graphs show subtraction images of temporal activity as recorded in group 1 . The upper graph shows right-sided activity minus left-sided activity and the lower TF-graph shows the resulting activity depending on the side of the stimulus (contralateral minus ipsilateral activity). The $10-30 \mathrm{~Hz}$ power values below also shows the resulting contralateral minus ipsilateral activity, in relation to the side of the stimulus. 


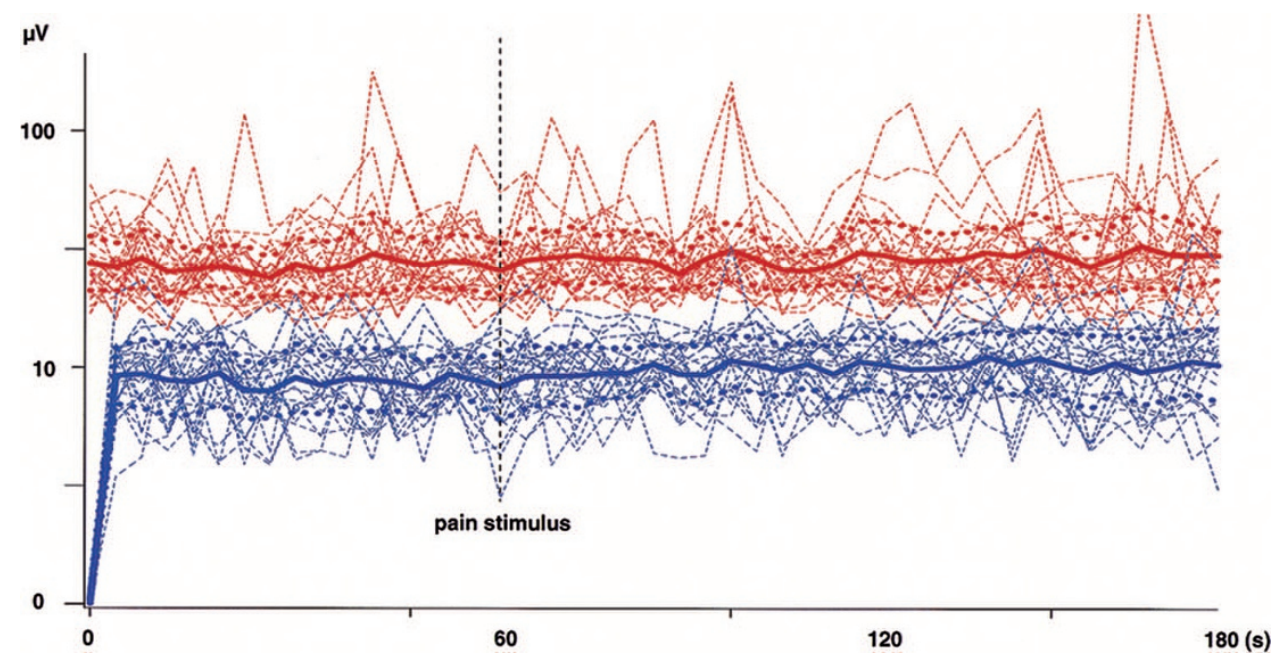

Figure 5. Response in amplitude-integrated EEG to a noxius stimulus. Individual traces of the upper and lower borders of the aEEG trend from group 3 are shown in thin lines, the thick lines depicts group means and the stippled lines the \pm SEM of the group mean. There is no consistent change in the aEEG trend after the pain stimulus. The amplitude scale on the $y$ axis is fully logarithmic (numbers indicate $\log$ value), as opposed to the standard aEEG output with a linear scale from 1 to 10 . This does not affect the absence of change in signal values.

\section{DISCUSSION}

Long-lasting and/or repetitive neonatal pain experiences can result in significant adverse long-term effects $(20,21)$. Development of reliable instruments for continuous assessment of pain in neonates with reduced ability to communicate pain would be a significant improvement in neonatal intensive care. Continuous EEG with the aEEG trend display is routinely used for monitoring of ill babies in many NICUs. An EEG-based monitor for assessing pain and/or distress would consequently be easy to implement in the NICU environment.

In the present study, painful stimuli induced changes (responses) in the EEG record which correlated with behavioral pain responses. However, these changes occurred mainly within the highest frequencies studied (i.e., $10-30 \mathrm{~Hz}$ ), they were not consistently lateralized, and they were more pronounced when the child was awake. Multiple lines of evidence strongly suggest that a major part of these responses comes from muscle activity rather than from cerebral cortex. First, the topography of the response was concentrated over the frontal and temporal areas where EEG recording is typically most contaminated by muscle activity. Second, spectral analysis demonstrated that the visually evident electromyogram (EMG) activity corresponded with the increase in the 10-30 $\mathrm{Hz}$ band after pain (Fig. 1). Third, the increase in this activity was correlated with a behavioral arousal of the infant. Fourth, we are not aware of any cortically generated EEG activity in any brain area during the neonatal period that would last so long. Finally, we are not aware of any situation where such long-standing (60-120 s) increase in beta activity of cortical origin would have been reported, neither spontaneously nor subsequent to any physiologic (i.e., nonpharmacologic) stimulus at any age.

Other studies have demonstrated that frontal EMG increases during painful stimulation and is an indicator of patient arousal. It has been reported that the frontal EMG may be of value even in assessing adequacy of anesthesia (22). A recent publication also demonstrates graded responses to pain stimulation associated with different depth of anesthesia (23). Further studies may clarify whether recording of facial or scalp muscle EMG activity is a useful adjunct also for neonatal pain monitoring.
A previous report in healthy newborns demonstrated an increase in frontal asymmetry in the theta band $(3-6 \mathrm{~Hz})$ as response to repeated "heel strokes," and its attenuation by sucrose before the procedure (10). The current study could not reproduce these results, and there may be several reasons for this. There were some differences in the details of stimulus procedures, which might have influenced the findings. To explore this further, we decided to perform an additional analysis with an open paradigm from the full signal (TF analysis; all channels).

This analysis shows that spontaneous fluctuation of EEG activity is partly independent between hemispheres, which challenges the analysis of asymmetry from relatively short EEG segments (also see Ref. 12).

In the study by Fernandez et al., a "heel stroke" was repeated four times every $15 \mathrm{~s}$ during $1 \mathrm{~min}$, whereas we only applied a moderate pressure during $1 \mathrm{~s}$ with a sharp tooth-pick. Whether this less painful procedure could explain the lack of EEG responses was addressed by evaluating EEG responses to routine blood sampling using two different techniques. Neither of them resulted in EEG asymmetry as response to the painful stimulus. It is possible that the different nature of the stimuli between the two studies may account for the differences in results, and that the stimulus applied by Fernandez et al. was more emotionally "unpleasant" than the relatively mild or painful stimuli, respectively, in the present study. Postnatal adaptation may also have affected the responses, because the infants in the study by Fernandez et al. were slightly older, around $1 \mathrm{wk}$, compared with the infants in the current study with a median age of just below 3 days $(71 \mathrm{~h})$. All infants in the study by Fernandez et al. were awake, whereas one third of the infants in the present study were asleep. Awake infants had increased responses, but there were no differences in frontal asymmetry related to sleep/wakefulness before the stimulus.

Emotional states have been correlated to frontal EEG asymmetry, as shown by increased left-sided activation (ie EEG suppression) in infants given sucrose as compared with infants given water (24) and it is well known that oral glucose reduces pain expression (25). We randomized the pin-prick infants to receive either $30 \%$ glucose according to pain relief recom- 
mendations (26) or water in a blinded fashion. There were no differences in EEG power measurements between the two treatments, although there was a significant difference in the PIPP scores. Sucrose, which was used in the study by Fernandez et al., may be slightly superior to glucose for pain relief during capillary heel sticks (27). However, this does not explain the lack of frontal asymmetry response to the stimulus.

In the current investigation, the infants who underwent blood sampling from the heel, displayed frontal EEG asymmetry with lower theta activity over the left side already before the stimulus. Left-sided EEG suppression was previously found in infants given sucrose, and in infants who did not cry when separated from their mothers, thus indicating a more positive emotional state $(11,24)$. Because we did not control for environmental factors in the two study centers, such differences cannot be excluded as explaining factors. Some differences between the centers may have occurred, such as the environment in group 3 was quieter, and consequently this could have resulted in a more positive emotional state in these infants.

This study was not designed to investigate a short (milliseconds) transient response to pain stimuli, i.e., short-latency event-related changes of the EEG activity. The aim was to assess possible EEG responses in infants who were transferred to another mental state as a result of the noxious stimulus. Such EEG activation might be of long latency and long lasting and could be associated with changes in cerebral blood flow. Hemodynamic responses to pain were evaluated by near infrared spectroscopy (NIRS) in newborn infants, an increase in oxygenated $\mathrm{Hb}$ was found over the central cortical regions $(28,29)$. Comparable to the results in the present study, awake subjects reacted more to the stimulus (29). Together with the findings by Fernandez et al., these studies suggest the presence of a transient and delayed ( $>10 \mathrm{~s}$ ) electrographic (EEG) or hemodynamic (NIRS) response to pain stimuli $(10,28,29)$.

In conclusion, this study shows that quantitative analysis of cortical brain activity by EEG is not a robust and useful method to evaluate pain or discomfort in newborn babies in a clinical context.

Acknowledgments. We thank RN Ann-Cathrine Berg and BMA Bodil Persson for recruiting the infants and performing the EEGs in Lund University hospital, and midwives and neonatal nurses in Lund and in Ålesund for assistance.

\section{REFERENCES}

1. Barker DP, Rutter N 1995 Exposure to invasive procedures in neonatal intensive care unit admissions. Arch Dis Child Fetal Neonatal Ed 72:F47-F48

2. Simons SH, van Dijk M, Anand KS, Roofthooft D, van Lingen RA, Tibboel D 2003 Do we still hurt newborn babies? A prospective study of procedural pain and analgesia in neonates. Arch Pediatr Adolesc Med 157:1058-1064

3. Anand KJ, Johnston CC, Oberlander TF, Taddio A, Lehr VT, Walco GA 2005 Analgesia and local anesthesia during invasive procedures in the neonate. Clin Ther 27:844-876

4. Stevens B, Johnston C, Gibbins S 2000 Pain Assessment in Neonates. Elsevier, Amsterdam, pp 101-134

5. Hummel P, van Dijk M 2006 Pain assessment: current status and challenges. Semin Fetal Neonatal Med 11:237-245

6. Anand KJ 2007 Pain assessment in preterm neonates. Pediatrics 119:605-607

7. Morison SJ, Grunau RE, Oberlander TF, Whitfield MF 2001 Relations between behavioral and cardiac autonomic reactivity to acute pain in preterm neonates. Clin J Pain 17:350-358

8. Taddio A, Shah V, Gilbert-MacLeod C, Katz J 2002 Conditioning and hyperalgesia in newborns exposed to repeated heel lances. JAMA 288:857-861

9. Brazelton TB 1995 Neonatal Behavioral Assessment Scale. Mac Keith Press, London

10. Fernandez M, Blass EM, Hernandez-Reif M, Field T, Diego M, Sanders C 2003 Sucrose attenuates a negative electroencephalographic response to an aversive stimulus for newborns. J Dev Behav Pediatr 24:261-266

11. Davidson RJ, Fox NA 1989 Frontal brain asymmetry predicts infants' response to maternal separation. J Abnorm Psychol 98:127-131

12. Hagemann D 2004 Individual differences in anterior EEG asymmetry: methodological problems and solutions. Biol Psychol 67:157-182

13. Stevens B, Johnston C, Petryshen P, Taddio A 1996 Premature infant pain profile: development and initial validation. Clin J Pain 12:13-22

14. Vanhatalo S, Palva JM, Andersson S, Rivera C, Voipio J, Kaila K 2005 Slow endogenous activity transients and developmental expression of $\mathrm{K}+-\mathrm{Cl}-$ cotransporter 2 in the immature human cortex. Eur J Neurosci 22:2799-2804

15. Vanhatalo S, Voipio J, Kaila K 2005 Full-band EEG (FbEEG): an emerging standard in electroencephalography. Clin Neurophysiol 116:1-8

16. Tolonen M, Palva JM, Andersson S, Vanhatalo S 2007 Development of the spontaneous activity transients and ongoing cortical activity in human preterm babies. Neuroscience 145:997-1006

17. Hellstrom-Westas L, Rosen I 2006 Continuous brain-function monitoring: state of the art in clinical practice. Semin Fetal Neonatal Med 11:503-511

18. Hellstrom-Westas LR, de Vries LS, Greisen G 2006 Amplitude-integrated EEG. classification and interpretation in preterm and term infants. NeoReviews 7:e76-e87

19. Rosen I 2006 The physiological basis for continuous electroencephalogram monitoring in the neonate. Clin Perinatol 33:593-611

20. Taddio A, Katz J, Ilersich AL, Koren G 1997 Effect of neonatal circumcision on pain response during subsequent routine vaccination. Lancet 349:599-603

21. Grunau RE, Holsti L, Peters JW 2006 Long-term consequences of pain in human neonates. Semin Fetal Neonatal Med 11:268-275

22. Tammisto T, Toikka O 1991 Spontaneous EMG activity for detection of arousal during general anaesthesia-comparison between recordings from frontal and neck musculature. Eur J Anaesthesiol 8:109-114

23. Wheeler P, Hoffman WE, Baughman VL, Koenig H 2005 Response entropy increases during painful stimulation. J Neurosurg Anesthesiol 17:86-90

24. Fox NA, Davidson RJ 1986 Taste-elicited changes in facial signs of emotion and the asymmetry of brain electrical activity in human newborns. Neuropsychologia 24:417-422

25. Eriksson M, Gradin M, Schollin J 1999 Oral glucose and venepuncture reduce blood sampling pain in newborns. Early Hum Dev 55:211-218

26. Larsson BA, Gradin M, Lind V, Selander B 2002 [Swedish guidelines for prevention and treatment of pain in the newborn infant]. Lakartidningen 99:1946-1949

27. Isik U, Ozek E, Bilgen H, Cebeci D 2000 Comparison of oral glucose and sucrose solutions on pain response in neonates. J Pain 1:275-278

28. Bartocci M, Bergqvist LL, Lagercrantz H, Anand KJ 2006 Pain activates cortical areas in the preterm newborn brain. Pain 122:109-117

29. Slater R, Cantarella A, Gallella S, Worley A, Boyd S, Meek J, Fitzgerald M 2006 Cortical pain responses in human infants. J Neurosci 26:3662-3666 